\title{
Approximations of closed-loop minimax MPC
}

\author{
Johan Löfberg \\ Division of Automatic Control \\ Department of Electrical Engineering \\ Linköpings universitet, SE-581 83 Linköping, Sweden \\ WWW: http://www. control.isy.liu.se \\ E-mail: johanl@isy.liu.se@isy.liu.se
}

April 22, 2003

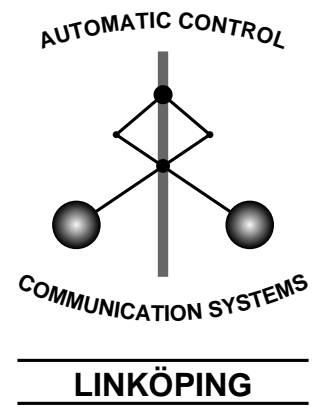

Report no.: LiTH-ISY-R-2516

Submitted to CDC03

Technical reports from the Control \& Communication group in Linköping are available at http://www. control.isy.liu.se/publications. 


\begin{abstract}
Minimax or worst-case approaches have been used frequently recently in model predictive control (MPC) to obtain control laws that are less sensitive to uncertainty. The problem with minimax MPC is that the controller can become overly conservative. An extension to minimax MPC that can resolve this problem is closed-loop minimax MPC. Unfortunately, closed-loop minimax MPC is essentially an intractable problem. In this paper, we introduce a novel approach to approximate the solution to a number of closed-loop minimax MPC problems. The result is convex optimization problems with size growing polynomially in system dimension and prediction horizon.
\end{abstract}

Keywords: Predictive Control, Optimal Control, Control of Uncertain Systems 


\title{
Approximations of closed-loop minimax MPC
}

\author{
Johan Löfberg \\ Department of Electrical Engineering \\ Linköping University, SE-581 83 Linköping, Sweden \\ WWW: http://www. control.isy.liu.se \\ Email: johanl@isy.liu.se
}

March 7, 2003

\begin{abstract}
Minimax or worst-case approaches have been used frequently recently in model predictive control (MPC) to obtain control laws that are less sensitive to uncertainty. The problem with minimax MPC is that the controller can become overly conservative. An extension to minimax MPC that can resolve this problem is closed-loop minimax MPC. Unfortunately, closed-loop minimax MPC is essentially an intractable problem. In this paper, we introduce a novel approach to approximate the solution to a number of closed-loop minimax MPC problems. The result is convex optimization problems with size growing polynomially in system dimension and prediction horizon.
\end{abstract}

\section{Minimax MPC}

The class of systems we address is linear discretetime systems with external disturbances

$$
\begin{aligned}
x_{k+1} & =A x_{k}+B u_{k}+G w_{k} \\
y_{k} & =C x_{k}
\end{aligned}
$$

The variables $x_{k} \in \mathbb{R}^{n}, u_{k} \in \mathbb{R}^{m}, y_{k} \in \mathbb{R}^{p}$ and $w_{k} \in \mathbb{R}^{r}$ denote the state, control input, controlled output and external disturbance respectively. Furthermore, the system is constrained, $u_{k} \in \mathbb{U}$ and $x_{k} \in \mathbb{X}$. The constraint sets $\mathbb{U}$ and $\mathbb{X}$ are assumed to be polyhedrons.

$$
\begin{aligned}
& u \in \mathbb{U}=\left\{u: E_{u} u \leq f_{u}\right\} \\
& x \in \mathbb{X}=\left\{x: E_{x} x \leq f_{x}\right\}
\end{aligned}
$$

The disturbance $w_{k}$ is only known to be bounded in some measure, but otherwise unknown. The set of possible disturbances is denoted $\mathbb{W}$.

$$
w_{k} \in \mathbb{W}
$$

The disturbance set $\mathbb{W}$ is one of the ingredients that determine the type of optimization problem we end up with. For simplicity, we concentrate on a box-constrained model.

$$
\mathbb{W}=\mathbb{W}_{\infty}=\left\{w:\|w\|_{\infty} \leq 1\right\}
$$

The dominating approach in robust MPC synthesis is to employ a minimax strategy, i.e., minimization of a worst-case performance measure. Openloop minimax MPC with a finite horizon performance measure can be stated as

$$
\begin{aligned}
u_{k} & =u_{k \mid k} \\
u_{\cdot \mid k} & =\min _{u} \max _{w} J_{k} \\
J_{k} & =\ell\left(x_{k \mid k}, u_{k \mid k}, \ldots, x_{k+N-1 \mid k}, u_{k+N-1 \mid k}\right)
\end{aligned}
$$

A fundamental flaw with this formulation is the fact that the MPC controller in reality applies feedback. This will make the minimax approach unnecessarily conservative, since it has to find a single control sequence that works well in open-loop for all admissible disturbance realizations.

In a closed-loop minimax MPC approach, one would assume instead that the future control $u_{k+1}$ is calculated optimally over the horizon $N-1$ first when $x_{k+1}$ is available. The problem to solve in closed-loop minimax MPC is thus

$$
\begin{aligned}
u_{\cdot \mid k} & =\min _{u_{k \mid k}} \max _{w_{k \mid k}} \cdots \min _{u_{k+N-1 \mid k}} \max _{w_{k+N-1 \mid k}} J_{k} \\
J_{k} & =\ell\left(x_{k \mid k}, u_{k \mid k}, \ldots, x_{k+N-1 \mid k}, u_{k+N-1 \mid k}\right)
\end{aligned}
$$


This type of minimax MPC has been addressed in, e.g., [7] and [12]. Unfortunately, the problems are intractable for anything but small systems and very short horizons, due to an exponential explosion in computational complexity. For example, the solution in [12] is based on a simple enumeration of the worst-case $\left(w_{k+j \mid k}= \pm 1\right)$ disturbance realizations, and a related enumeration of the possible control and state trajectories. See Figure 1.

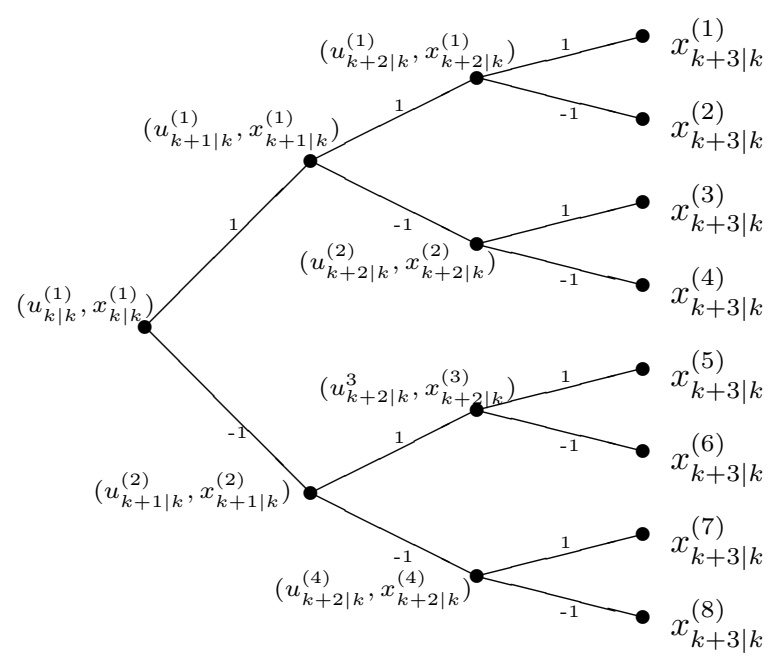

Figure 1: The figure shows the exponential explosion in the number of disturbance realizations and corresponding control sequences that have to be defined to solve closed-loop minimax MPC using straightforward enumeration.

To solve the closed-loop minimax problem, one introduces decision variables $u_{\cdot \mid k}^{(i)}$, and related state realization $x_{\cdot \mid k}^{(i)}$, for each possible disturbance realization $w_{\cdot \mid k}^{(i)}$, and solves

$$
\begin{aligned}
\min _{t, u_{\cdot k}^{(i)}} t & \\
\text { s. t. } \ell\left(x_{k \mid k}^{(i)}, u_{k \mid k}, \ldots, x_{k+N-1 \mid k}^{(i)}, u_{k+N-1 \mid k}^{(i)}\right) & \leq t \\
x_{k+j \mid k}^{(i)} & \in \mathbb{X} \\
u_{k+j \mid k}^{(i)} & \in \mathbb{U}
\end{aligned}
$$

Explicit off-line solutions are possible in some cases $[2,6]$, but the exponential complexity remain, although now in the off-line computations.

\section{Parameterization of $U$}

A standard trick to reduce conservatism in openloop minimax schemes is feedback predictions $[1$, $11,4]$. The idea is to assume that at least some feedback will be employed. This can be done by parameterizing the future control sequence in terms of the future states and a new decision variable $v_{k+j \mid k} \in \mathbb{R}^{m}$,

$$
u_{k+j \mid k}=L x_{k+j \mid k}+v_{k+j \mid k}
$$

The feedback matrix $L$ is typically chosen off-line, or some heuristic procedure is used to find a suitable $L$ on-line. Anyway, $L$ is fixed in the actual solution of the minimax problem.

For compact notation, introduce stacked vectors with future outputs, states, control inputs and disturbances

$$
\begin{aligned}
Y & =\left(\begin{array}{llll}
y_{k \mid k}^{T} & y_{k+1 \mid k}^{T} & \cdots & y_{k+N-1 \mid k}
\end{array}\right)^{T} \\
X & =\left(\begin{array}{llll}
x_{k \mid k}^{T} & x_{k+1 \mid k}^{T} & \cdots & x_{k+N-1 \mid k}
\end{array}\right)^{T} \\
U & =\left(\begin{array}{llll}
u_{k \mid k}^{T} & u_{k+1 \mid k}^{T} & \cdots & u_{k+N-1 \mid k}^{T}
\end{array}\right)^{T} \\
V & =\left(\begin{array}{llll}
v_{k \mid k}^{T} & v_{k+1 \mid k}^{T} & \cdots & v_{k+N-1 \mid k}^{T}
\end{array}\right)^{T} \\
W & =\left(\begin{array}{llll}
w_{k \mid k}^{T} & w_{k+1 \mid k}^{T} & \cdots & w_{k+N-1 \mid k}^{T}
\end{array}\right)^{T} \\
\mathbb{W}^{N} & =\mathbb{W} \times \mathbb{W} \cdots \times \mathbb{W}
\end{aligned}
$$

The predicted states and outputs, and the control and state constraints, can be written as

$$
\begin{aligned}
Y & =\mathcal{C} X \\
X & =\mathcal{A} x_{k \mid k}+\mathcal{B} U+\mathcal{G} W \\
\mathcal{E}_{x}\left(\mathcal{A} x_{k \mid k}+\mathcal{B} U+\mathcal{G} W\right) & \leq \mathcal{F}_{x} \\
\mathcal{E}_{u} U & \leq \mathcal{F}_{u}
\end{aligned}
$$

where $\mathcal{A}, \mathcal{B}, \mathcal{G}, \mathcal{C} \mathcal{E}_{x}, \mathcal{F}_{x}, \mathcal{E}_{u}$ and $\mathcal{F}_{u}$ follow easily from (1) and (2).

Feedback prediction in the form (7) can be written as

$$
U=\mathcal{L}_{X} X+V
$$

where $\mathcal{L}_{X}=\oplus_{j=1}^{N} L$. Closing the loop gives the following predictions $\left(\Omega=\left(I-\mathcal{B} \mathcal{L}_{X}\right)^{-1}\right)$

$$
\begin{aligned}
X & =\Omega\left(\mathcal{A} x_{k \mid k}+\mathcal{B} V+\mathcal{G} W\right) \\
U & =\mathcal{L}_{X} \Omega\left(\mathcal{A} x_{k \mid k}+\mathcal{B} V+\mathcal{G} W\right)+V
\end{aligned}
$$


The problem is that the mapping from $\mathcal{L}$ and $V$ to $X$ and $U$ is nonlinear, hence optimization over both $\mathcal{L}_{X}$ and $V$ is likely to cause problem. At least, it is not obvious how this parameterization can be incorporated in a standard convex optimization problem.

Let us look at bit closer on the parameterized control sequence.

$$
U=\left(\mathcal{L}_{X} \Omega\left(\mathcal{A} x_{k \mid k}+\mathcal{B} V\right)+V\right)+\mathcal{L}_{X} \Omega \mathcal{G} W
$$

It is composed of one certain part, $\mathcal{L}_{X} \Omega\left(\mathcal{A} x_{k \mid k}+\right.$ $\mathcal{B} V)+V$, and one mapping from the disturbances to the control sequence, $\mathcal{L}_{X} \Omega \mathcal{G} W$. Important to remember is that $\mathcal{L}_{X} \Omega \mathcal{G}$ has a causal structure, i.e. $u_{k+j \mid k}$ depend only on $w_{k+i \mid k}, i<j$.

The findings motivate us to try an alternative parameterization.

$$
\begin{aligned}
U & =\mathcal{L} W+V \\
\mathcal{L} & =\left(\begin{array}{ccc}
0 & 0 & \cdots \\
L_{10} & 0 & \cdots \\
L_{20} & L_{21} & \cdots \\
\vdots & \vdots & \ddots \\
L_{(N-1) 0} & L_{(N-1) 1} & L_{(N-1)(N-2)}
\end{array}\right.
\end{aligned}
$$

The control sequence is now parameterized directly in the uncertainty. The matrix $L_{i j} \in \mathbb{R}^{m \times r}$ describes how $u_{k+i \mid k}$ uses $w_{k+j \mid k}$. Note that the parameterization is causal in the same sense as the standard parameterization (10). Inserting the parameterization yields

$$
\begin{aligned}
X & =\mathcal{A} x_{k \mid k}+\mathcal{B} V+(\mathcal{G}+\mathcal{B} \mathcal{L}) W \\
U & =\mathcal{L} W+V
\end{aligned}
$$

The mapping from $\mathcal{L}$ and $V$ to $X$ and $U$ is now bilinear. This is the main idea in this work, and it will allow us to formulate a number of convex minimax MPC problems (with polynomial complexity in the prediction horizon $N$ and system dimensions).

\section{Minimax MPC is Convex in $\mathcal{L}$ and $V$}

In the previous section, a parameterization of the future control sequence was proposed. It will now be shown that this parameterization allows us to solve some minimax MPC problems with on-line optimization of feedback predictions using convex programming.

\subsection{Minimum Peak Performance}

A commonly used performance measure in minimax MPC is minimization of worst-case deviation along the predicted trajectory $[3,14,10]$, the minimum peak problem. Of course, the results here can be generalized to deviation from a reference trajectory, but this is omitted to keep notation simple. The problem can be stated as

$$
\begin{array}{crll}
\min _{u} \max _{w} \max _{j} & \left\|y_{k+j \mid k}\right\|_{\infty} & \\
\text { s.t. } & u_{k+j \mid k} \in \mathbb{U} \forall w \in \mathbb{W} \\
& x_{k+j \mid k} \in \mathbb{X} \forall w \in \mathbb{W} \\
& w_{k+j \mid k} \in \mathbb{W} &
\end{array}
$$

Rewrite to a compact epigraph formulation by noting that $\max _{j}\left\|y_{k+j \mid k}\right\|_{\infty}=\|Y\|_{\infty}$. Furthermore, introduce the proposed feedback predictions and vectorize control and state constraints.

$\min _{V, \mathcal{L}, t} t$

$$
\begin{aligned}
\text { s. t. }|| \mathcal{C}\left(\mathcal{A} x_{k \mid k}+\mathcal{B} V+(\mathcal{G}+\mathcal{B} \mathcal{L}) W\right) \|_{\infty} & \leq t \\
\mathcal{E}_{u}(V+\mathcal{L} W) & \leq \mathcal{F}_{u} \\
\mathcal{E}_{x}\left(\mathcal{A} x_{k \mid k}+\mathcal{B} V+(\mathcal{G}+\mathcal{B} \mathcal{L}) W\right) & \leq \mathcal{F}_{x}
\end{aligned}
$$

The peak constraint is equivalent to two sets of linear inequalities

$$
\begin{aligned}
& \mathcal{C}\left(\mathcal{A} x_{k \mid k}+\mathcal{B} V\right)+\mathcal{C}(\mathcal{G}+\mathcal{B} \mathcal{L}) W \leq t \quad \forall W \in \mathbb{W}^{N} \\
& -\mathcal{C}\left(\mathcal{A} x_{k \mid k}+\mathcal{B} V\right)-\mathcal{C}(\mathcal{G}+\mathcal{B} \mathcal{L}) W \leq t \quad \forall W \in \mathbb{W}^{N}
\end{aligned}
$$

To satisfy these uncertain linear inequalities, we need to maximize the elements in the vector $\mathcal{B L}) W$. It can easily be shown that $\max _{|x| \leq 1} P x=|P| \mathbf{1}$. Hence, the uncertain constraints are satisfied if

$$
\begin{aligned}
\mathcal{C}\left(\mathcal{A} x_{k \mid k}+\mathcal{B} V\right)+|\mathcal{C}(\mathcal{G}+\mathcal{B} \mathcal{L})| \mathbf{1} & \leq t \\
-\mathcal{C}\left(\mathcal{A} x_{k \mid k}+\mathcal{B} V\right)+|\mathcal{C}(\mathcal{G}+\mathcal{B} \mathcal{L})| \mathbf{1} & \leq t
\end{aligned}
$$

To take care of these constraints using linear programming, we need to bound the absolute value of the matrix $\mathcal{C}(\mathcal{G}+\mathcal{B} \mathcal{L})$ from above. This is done by defining a matrix variable $\Omega$

$$
\begin{aligned}
\mathcal{C}(\mathcal{G}+\mathcal{B L}) & \leq \Omega \\
-\mathcal{C}(\mathcal{G}+\mathcal{B L}) & \leq \Omega
\end{aligned}
$$


and the peak constraint is equivalent to

$$
\begin{aligned}
\mathcal{C}\left(\mathcal{A} x_{k \mid k}+\mathcal{B} V\right)+\Omega \mathbf{1} & \leq t \\
-\mathcal{C}\left(\mathcal{A} x_{k \mid k}+\mathcal{B} V\right)+\Omega \mathbf{1} & \leq t
\end{aligned}
$$

The same method can be applied to state and control constraints and gives a matrix variable $\Upsilon$ and the constraints

$$
\begin{aligned}
\left(\begin{array}{c}
\mathcal{E}_{x}\left(\mathcal{A} x_{k \mid k}+\mathcal{B} V\right) \\
\mathcal{E}_{u} V
\end{array}\right)+\Upsilon \mathbf{1} & \leq\left(\begin{array}{c}
\mathcal{F}_{x} \\
\mathcal{F}_{u}
\end{array}\right) \\
\left(\begin{array}{c}
\mathcal{E}_{x}(\mathcal{G}+\mathcal{B} \mathcal{L}) \\
\mathcal{E}_{u} \mathcal{L}
\end{array}\right) & \leq \Upsilon \\
-\left(\begin{array}{c}
\mathcal{E}_{x}(\mathcal{G}+\mathcal{B} \mathcal{L}) \\
\mathcal{E}_{u} \mathcal{L}
\end{array}\right) & \leq \Upsilon
\end{aligned}
$$

To summarize, the minimum peak problem with element-wise bounded disturbances and feedback predictions is solved with the following linear program.

$$
\begin{aligned}
& \min _{U, \mathcal{L}, t, \Omega, \Upsilon} t \\
& \text { s. t. } \\
& \begin{aligned}
& \mathcal{C}\left(\mathcal{A} x_{k \mid k}+\mathcal{B} V\right)+\Omega 1 \leq t \\
&-\mathcal{C}\left(\mathcal{A} x_{k \mid k}+\mathcal{B} V\right)+\Omega 1 \leq t \\
& \mathcal{C}(\mathcal{G}+\mathcal{B} \mathcal{L}) \leq \Omega \\
&-\mathcal{C}(\mathcal{G}+\mathcal{B} \mathcal{L}) \leq \Omega \\
&\left(\mathcal{E}_{x}\left(\mathcal{A} x_{k \mid k}+\mathcal{B} V\right)\right. \\
&\left.\mathcal{E}_{u} V\right)+\Upsilon 1 \leq\left(\begin{array}{c}
\mathcal{F}_{x} \\
\mathcal{F}_{u}
\end{array}\right)
\end{aligned} \\
& \left(\begin{array}{c}
\mathcal{E}_{x}(\mathcal{G}+\mathcal{B} \mathcal{L}) \\
\mathcal{E}_{u} \mathcal{L}
\end{array}\right) \leq \Upsilon \\
& -\left(\begin{array}{c}
\mathcal{E}_{x}(\mathcal{G}+\mathcal{B} \mathcal{L}) \\
\mathcal{E}_{u} \mathcal{L}
\end{array}\right) \leq \Upsilon
\end{aligned}
$$

The derived optimization problem can be solved with a standard linear programming solver. This means that the introduction of $\mathcal{L}$ as a free variable has not complicated the problem in terms of conceptual complexity. However, the number of introduced variables and constraints is huge. This will be discussed further in Section 4 .

\subsection{Quadratic Performance}

The second minimax MPC problem we address is a formulation with the standard quadratic performance measure $(Q, R \succ 0)$.

$$
\begin{aligned}
\min _{u} \max _{w} & \sum_{j=0}^{N-1}\left\|y_{k+j \mid k}\right\|_{Q}^{2}+\left\|u_{k+j \mid k}\right\|_{R}^{2} \\
\text { s.t. } & u_{k+j \mid k} \in \mathbb{U} \forall w \in \mathbb{W} \\
& x_{k+j \mid k} \in \mathbb{X} \forall w \in \mathbb{W} \\
& w_{k+j \mid k} \in \mathbb{W}
\end{aligned}
$$

This is an intractable problem, since it involves maximization of a convex quadratic function. However, it has recently been shown by the author that semidefinite relaxations can be employed to solve a conservative approximation [9]. To this end, introduce $\mathcal{Q}=\oplus_{j=1}^{N} Q$ and $\mathcal{R}=\oplus_{j=1}^{N} R$. The minimax problem with feedback predictions can be written as the following uncertain program

$$
\begin{array}{lrl}
\min _{V, \mathcal{L}, t} t & \\
\text { s. t. } & Y^{T} \mathcal{Q} Y+U^{T} \mathcal{R} U & \leq t \forall W \in \mathbb{W}^{N} \\
\mathcal{E}_{u}(V+\mathcal{L} W) & \leq \mathcal{F}_{u} \forall W \in \mathbb{W}^{N} \\
\mathcal{E}_{x}\left(\mathcal{A} x_{k \mid k}+\mathcal{B} V+(\mathcal{G}+\mathcal{B} \mathcal{L}) W\right) & \leq \mathcal{F}_{x} \forall W \in \mathbb{W}^{N}
\end{array}
$$

Robust semidefinite programming theory [5] can be used to guarantee the quadratic performance constraint. Introduce, for notational simplicity, $Y_{V}=\left(\mathcal{C}\left(\mathcal{A} x_{k \mid k}+\mathcal{B} V\right)\right)^{T}$ and $\mathcal{D}=\mathcal{C}(\mathcal{G}+\mathcal{B} \mathcal{L})$. The performance constraint holds if there exists a multiplier $\tau \in \mathbb{R}_{+}^{N r}$ and a diagonal matrix $\mathcal{T}=\oplus_{j=1}^{N r} \tau_{j}$ such that the following matrix inequality is feasible (see [9] for details and the derivation)

$$
\left(\begin{array}{cccc}
t-\mathbf{1}^{T} \tau & Y_{V}^{T} & V^{T} & 0 \\
\star & \mathcal{Q}^{-1} & 0 & \mathcal{D} \\
\star & 0 & \mathcal{R}^{-1} & \mathcal{L} \\
0 & \star & \star & \mathcal{T}
\end{array}\right) \succeq 0
$$

Note that the semidefinite relaxation of the minimax problem is linear in $V, \mathcal{L}$ and $\tau$.

Linear state and control constraint can be taken care of using the methods described in the previous section, i.e., the problem will only be augmented with a set of linear inequalities. Our solution to the minimax problem is thus given by the following semidefinite program.

$$
\begin{aligned}
\min _{V, \mathcal{L}, t, \Omega, \Upsilon, \tau} & t \\
\text { s. t. } & (18),(19)
\end{aligned}
$$

\section{Cheaper Parameterizations}

The main problem with the proposed minimax formulations is the excessive amount of decision variables and constraints.

The reason is, to begin with, the highdimensional parameterization of the matrix $\mathcal{L}$. The number of free variables in this matrix alone is

$m r((N-1)+(N-2)+\ldots+1)=\frac{m r}{2} N(N-1)$ 
In addition to this, the matrices $\Omega$ and $\Upsilon$ also introduce $O\left(N^{2}\right)$ variables.

Note however that many of the variables and constraints are redundant. For instance, the matrix $\mathcal{C}(\mathcal{G}+\mathcal{B} \mathcal{L})$ is lower block triangular, so $\Omega$ can also be lower block triangular. These issues will not be dealt with here. The software used to implement the algorithms, YALMIP [8] and SEDUMI [13], take care of redundant variables and constraints automatically.

Without going into details, it can be shown that the standard parameterization (10) can be recovered using only $O(N)$ variables. The reason is the inherent shift property of linear time-invariant systems. Essentially, $w_{k}$ affects $x_{k+j}$ in the same way as $w_{k+i}$ affects $x_{k+j+i}$. This implies that the matrix $\mathcal{L}_{X} \Omega \mathcal{G}$ has a lower block diagonal Toeplitz structure. For details, and other ways to reduce complexity, see [9].

\section{Simulation Results}

\section{Example 1 (Unstable third-order system)}

The system is given by ${ }^{1}$

$$
\begin{aligned}
A & =\left(\begin{array}{ccc}
2.938 & -0.7345 & 0.25 \\
4 & 0 & 0 \\
0 & 1 & 0
\end{array}\right), B=\left(\begin{array}{c}
0.25 \\
0 \\
0
\end{array}\right) \\
G & =\left(\begin{array}{c}
0.0625 \\
0 \\
0
\end{array}\right) \\
C & =\left(\begin{array}{lll}
-0.2072 & 0.04141 & 0.07256
\end{array}\right) x_{k}
\end{aligned}
$$

The control objective is to keep the output $y_{k}$ as close as possible to the reference value 1 , but at the same time never exceed this level ${ }^{2}$. The input is constrained $\left|u_{k}\right| \leq 1$. Since the main objective is to balance the output close to the constraint level, the weight matrices are chosen as $Q=1$ and $R=0.01$. The prediction horizon was $N=10$. The proposed minimax controller with free feedback predictions was implemented, together with a number of minimax controllers ${ }^{3}$ using parameterization (10) with

\footnotetext{
${ }^{1}$ Discretized version (zero-order hold, sample-time 1 second) of the system $\frac{0.25^{2}(-2 s+1)}{s\left(s^{2}+0.25^{2}\right)}$.

${ }^{2}$ The extension of the proposed minimax controller to reference tracking is straightforward and the details are omitted for brevity. The interested reader is referred to [9]

${ }^{3}$ See [9] for details
}

fixed feedback matrices defined using $L Q$ controllers with control weights ranging from $10^{-3}$ to $10^{4}$. The results are given in Figure 2. The performance is

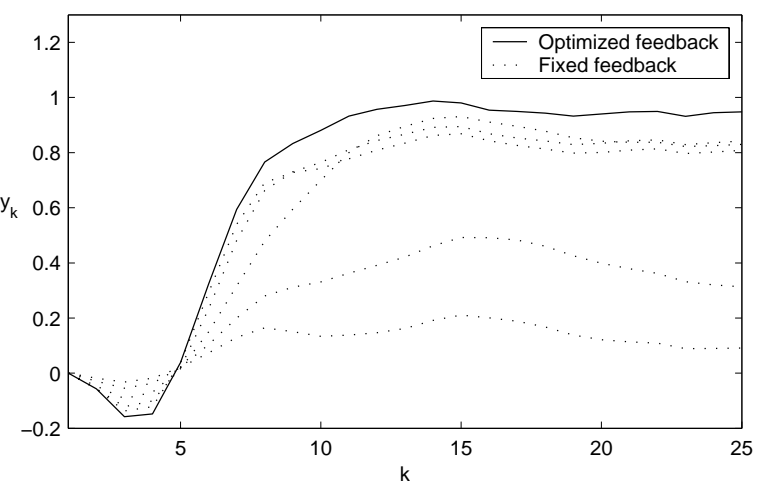

Figure 2: Closed-loop response with the proposed parameterization of the feedback prediction. Dashed lines represent closed-loop responses from minimax controllers using fixed feedback matrices defined using LQ controllers with different control weights ranging from $10^{-3}$ to $10^{4}$

substantially improved with the proposed minimax controller. The output $y_{k}$ is stationed around 0.95, while the best minimax controller with a fixed feedback matrix settles at an output level around 0.85 .

The ultimate test is to see how the proposed controller compares to the exact solution to the closedloop minimax MPC problem. Define the extreme realizations $W \in \mathbf{C o}\left\{W^{(1)}, W^{(2)}, \ldots, W^{\left(2^{N-1}\right)}\right\}$ and corresponding control realizations $U^{(i)}$ and state realizations $X^{(i)}=\mathcal{A} x_{k \mid k}+\mathcal{B} U^{(i)}+\mathcal{G} W^{(i)}$ (see Figure 1). The closed-loop minimax problem, applied to our quadratic performance measure, can be written as ${ }^{4}$.

$$
\begin{array}{|rrl}
\hline \min _{U^{i}, t} t & \\
\text { s. t. } & \left\|\mathcal{Q}^{1 / 2} \mathcal{C} X^{(i)}\right\| & \leq t \\
& \left\|\mathcal{R}^{1 / 2} U^{(i)}\right\| & \leq \\
& \mathcal{E}_{u} U^{(i)} & \leq \mathcal{F}_{u} \\
& \mathcal{E}_{x} X^{(i)} & \leq \mathcal{F}_{x}
\end{array}
$$

Let us implement this exact solution and compare it to the proposed minimax controller.

\footnotetext{
${ }^{4}$ The problem is written as a second order cone problem to enable use of SEDUMI
} 


\section{Example 2 (Comparison with exact solution) References}

All numerical data is the same as in the previous example, except for the prediction horizon $N$ which now decreased to $N=7$. The reason is the exponential explosion of the number of variables and constraints in the exact closed-loop minimax solution. Step-responses for the proposed minimax controller and an exact closed-loop minimax MPC controller are given in Figure 3.

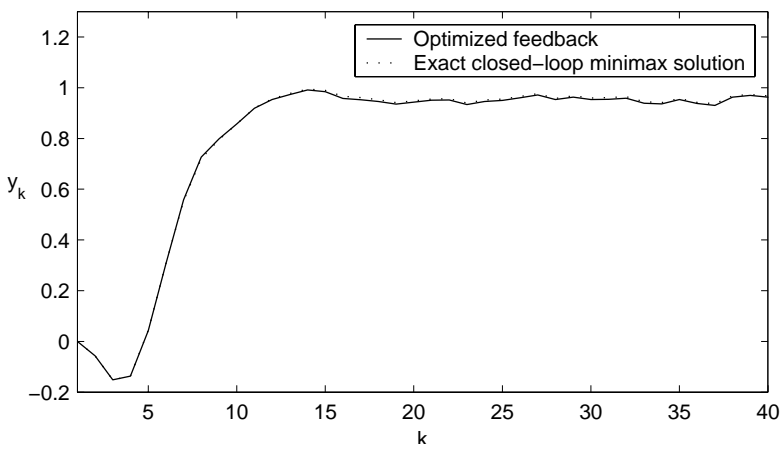

Figure 3: Step-responses for proposed minimax controller with on-line optimized feedback predictions, and the exact closed-loop minimax MPC controller. Closed-loop behavior is essentially the same.

As one can see in the figure, the performance of the two controllers is almost identical. The results are very encouraging, considering that two approximations are involved in our minimax controller (the semidefinite relaxation and the linear parameterization of $U$ ).

\section{Conclusion}

A novel approach to improve conservativiness in minimax MPC has been proposed. A new parameterization of the control sequence allows approximation of closed-loop minimax MPC using standard convex programming. The convex programs scale reasonably well with problem dimensions. Numerical experiments indicates that the performance is substantially improved compared to standard minimax schemes, and is comparable with the exact closed-loop minimax MPC solution.
[1] A. Bemporad. Reducing conservativeness in predictive control of constrained systems with disturbances. In Proceedings of 37th Conference on Decision and Control, pages 1384-1389, Tampa, Florida, 1998.

[2] F. Borrelli. Discrete Time Constrained Optimal Control. PhD thesis, Swiss Federal Institute of Technology (ETH), 2002.

[3] P. J. Campo and M. Morari. Robust model predictive control. In Proceedings of the American Control Conference, pages 1021-1025, Minneapolis, Minnesota, 1987.

[4] L. Chisci, P. Falugi, and G. Zappa. Predictive control for constrained systems with polytopic uncertainty. In Proceedings of the American Control Conference 2001 , Arlington, Virginia, June 2001.

[5] L. El Ghaoui, F. Oustry, and H. Lebret. Robust solutions to uncertain semidefinite programs. SIAM Journal on Optimization, 9(1):33-52, 1998.

[6] E. C. Kerrigan and J. M. Maciejowski. Feedback min-max model predictive control using a single linear program: Robust stability and the explicit solution. Technical Report CUED/F-INFENG/TR.440, Departement of Engineering, University of Cambridge, 2002.

[7] J. H. Lee and Z. Yu. Worst-case formulations of model predictive control for systems with bounded parameters. Automatica, 33(5):763-781, 1997.

[8] J. Löfberg. YALMIP 2.2 - User's Guide. Linköpings universitet, Sweden, 2002.

[9] J. Löfberg. Minimax approaches to robust model predictive control. PhD thesis, Linköpings universitet, 2003.

[10] G. H. C Oliviera, W. C. Amaral, G. Favier, and G. A. Dumont. Constrained robust predictive controller for uncertain processes modeled by orthonormal series functions. Automatica, 36(4):563-572, 2000.

[11] J. Schuurmans and J. A. Rossiter. Robust predictive control using tight sets of predicted states. Control Theory and Applications, 147(1):13-18, 2000.

[12] P. O. M. Scokaert and D. Q. Mayne. Min-max feedback model predictive control for constrained linear systems. IEEE Transactions on Automatic Control, 43(8):11361142, 1998.

[13] J. F. Sturm. Using SeDuMi 1.02, a MATLAB toolbox for optimization over symmetric cones. Optimization Methods and Software, 11-12(1-4):625-653, 1999.

[14] A. Zheng. Robust Control of Systems Subject to Constraints. PhD thesis, California Institute of Technology, Pasadena, California, 1995. 